\title{
Combined mucinous cystadenoma and carcinoid tumour of the appendix with coexistent features of an endometrioma: a case report
}

\begin{abstract}
Introduction: Endometriosis is defined as 'the presence of endometrial glands and stroma outside the uterine cavity'. Appendiceal cancers are rare tumours of the gastrointestinal tract. There are some reported cases of these two disease processes occurring simultaneously within separate lesions. However, there are no reported cases of the two diseases occurring within the same entity. We report the unique case of an appendiceal cancer (combined mucinous cyst adenoma and carcinoid tumour) with coexistant histological features of an endometrioma.
\end{abstract}

Case report: A 36 year old nulliparous woman was referred to clinic with primary infertility, with an unremarkable past medical history. After routine infertility investigations, diagnostic laparoscopy was carried out and endometriosis was diagnosed. A suspicious lesion was also seen on the appendix. A right hemicolectomy and appendectomy was performed. Histological results showed mucinous cystadenoma with well differentiated carcinoid tumour and coexistent features of an endometrioma.

Discussion: An association between endometriosis and cancer has been well documented in literature. Existence with appendiceal cancers is rare. Carcinoids and cyst adenomas are both common types of appendiceal cancers, found incidentally or mimicking acute appendicitis. Occurrence in the same lesion is rare and unique to be found with features of an endometrioma.

Conclusion: This case illustrates the broad spectrum of appendiceal and endometrial disease. We hope to highlight the interesting asymptomatic presentation of this patient and therefore the importance of requesting routine histopathological analysis after appendicectomy.

Keywords: endometrioma, appendix, mucinouscystadenoma
Volume I Issue I - 2014

\author{
Suzanna Dunkerton,' Neena K Pankhania, \\ Chris Johnson, ${ }^{2}$ Terek Gelbaya' \\ 'Gynaecology, Leicester Royal Infirmary, UK \\ 2Pathology, Leicester Royal Infirmary, UK
}

Correspondence: Suzanna Dunkerton, Leicester General Hospital, Leicester, Leics, UK, Tel 07979591333 , Emailslaird@doctors.org.uk

Received: August 15, 2014 | Published: August 20, 2014

\section{Introduction}

Endometriosis is defined as the presence of endometrial glands and stroma outside the uterine cavity ${ }^{1,2}$ and is thought to affect up to $10 \%$ of women. ${ }^{3}$ Endometriotic lesions can be found anywhere in the body, common sites are the ovaries, pelvic peritoneum and fallopian tubes. It is less commonly found at the cervix, bladder, lungs and bowel. ${ }^{4}$

An association between endometriosis and malignancy has been well documented in literature despite reported controversy of the relationship. ${ }^{5,6}$ Brinton et al. ${ }^{7}$ evaluated a large cohort of Swedish women $(20,868)$ with a diagnosis of endometriosis, were an increased risk of cancer was found; in particular ovarian, breast and haematopoietic malignancies. Reports of endometriosis associated with cancers of the bowel are rare.

Appendicular tumours are rare and account for only $0.4 \%$ of all gastrointestinal tumours. ${ }^{8}$ They are usually found incidentally or during investigations for other disease processes (as in our case), accounting for $1 \%$ of appendectomies. ${ }^{9}$

Reports of endometriosis associated with bowel cancer are rare. One similar case has been reported by Azordgean et al., ${ }^{10}$ which documented the coexistence of a carcinoid tumour of the appendix and ileal endometriosis, each found at separate locations. ${ }^{10}$ This case presented with right lower abdominal pain mimicking acute appendicitis and was treated surgically with excision of the ileum and appendix. We describe the unique, not yet reported case of a 36 year old woman presenting with primary infertility, who was found to have a mucinous cyst-adenoma and carcinoid tumour of the appendix with co-existent endometriosis.

\section{Case presentation}

A 36 year old asymptomatic woman was referred with a 3 year history of primary infertility. Her periods occurred every 35-38 days with an average bleed and she engaged in regular unprotected coitus. No other personal or family medical history was noted and she led a healthy lifestyle as a non-smoker with minimal alcohol intake.

Routine infertility tests for herself and her partner were mostly normal. Except her ultrasound scan revealed 3-4 endometriotic cysts in the right ovary, the largest being $3.5 \mathrm{~cm}$ in diameter with a small cyst also found in the left ovary. Endocervical swabs revealed Chlamydia which was promptly treated. It was decided to proceed with a diagnostic laparoscopy with tubal patency dye test and excision of endometriosis if appropriate. 
Diagnostic laparoscopy found extensive uterovesical, peritoneal and ovarian endometriosis.

Figure 1: Right lateral bowel adhesions were dissected and endometriomas were excised from both ovaries and were mobilised as per RCOG guidelines. ${ }^{2}$ The dye test revealed the left tube was patent and the right blocked. Overall, grade 4 endometriosis was noted. On further visualisation of the abdomen, a suspicious looking mucoid lesion on the tip of the appendix was seen and biopsied.

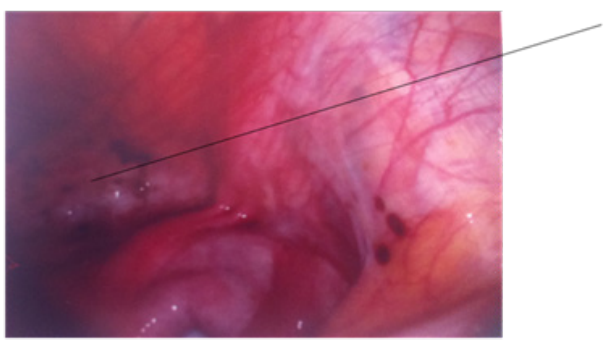

Endometriosis

Figure I Endometriosis seen at initial laparoscopy.

Figure 2: Histology of the appendix tip showed mucinous material within the bowel wall and stroma suggesting a mucocele or mucinous tumour of the appendix. The patient was referred to lower gastrointestinal surgeons. A CT scan found it difficult to visualise the appendix but adjacent to the caecum a $10 \mathrm{~mm}$ low attenuation lesion with mural calcification was noted suggestive of an appendicular mucocele. No pelvic or retroperitoneal lymphadenopathy was noted and no lesions were noted within the chest.

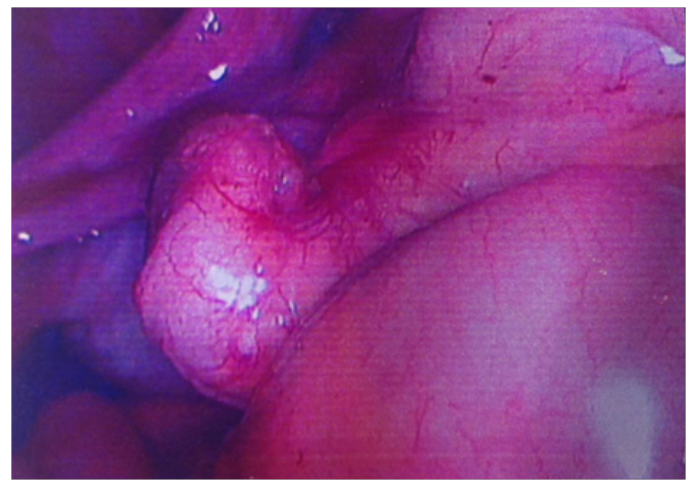

Figure 2 Histology of the appendix tip showed mucinous material within the bowel wall and stroma suggesting a mucocele or mucinous tumour of the appendix.

Figure 3: The patient underwent a right hemicolectomy during which further endometriotic deposits were seen. The appendix appeared abnormal as it was thickened and adherent to caecum with mucinous material extruding from the tip. Three proximal puckered lesions were noted in last $20 \mathrm{~cm}$ of the terminal ileum but no other peritoneal disease was noted.

Histological results found the same appendix showed.

i) Mucinous cystadenoma of the appendix

ii) Well differentiated carcinoid tumour measuring $10 \mathrm{~mm}$ in diameter

iii) Coexistent features of endometriosis
Figure 4 \& Figure 5: Currently, the patient is well and conceived naturally whilst waiting to start IVF treatment.

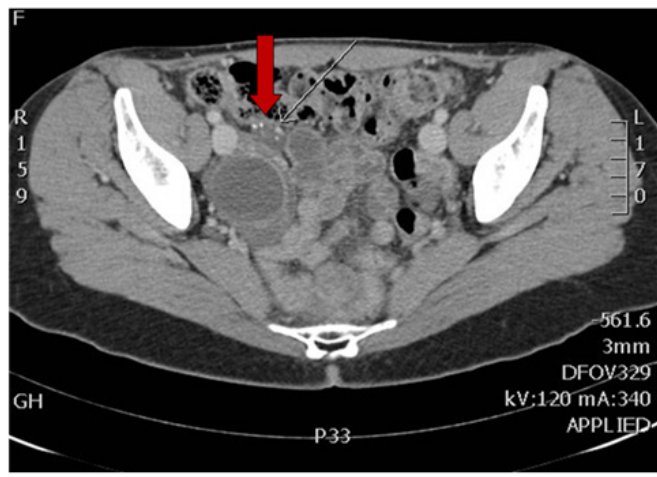

Figure $3 \mathrm{CT}$ scan. Adjacent to the caecum a $10 \mathrm{~mm}$ low attenuation lesion with mural calcification was noted suggestive of an appendicular mucocele.

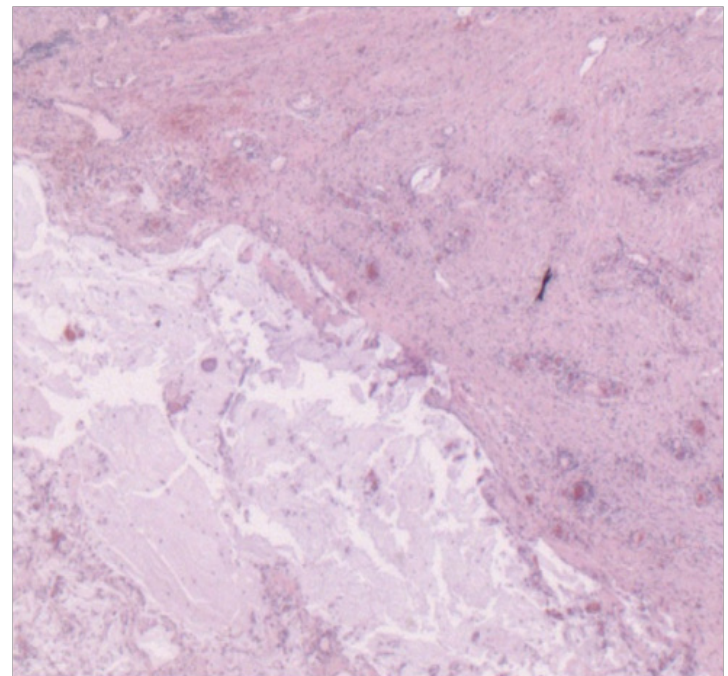

Figure 4 Appendis take from hemicolectomy specimen, showing mucin on the serosal surface.

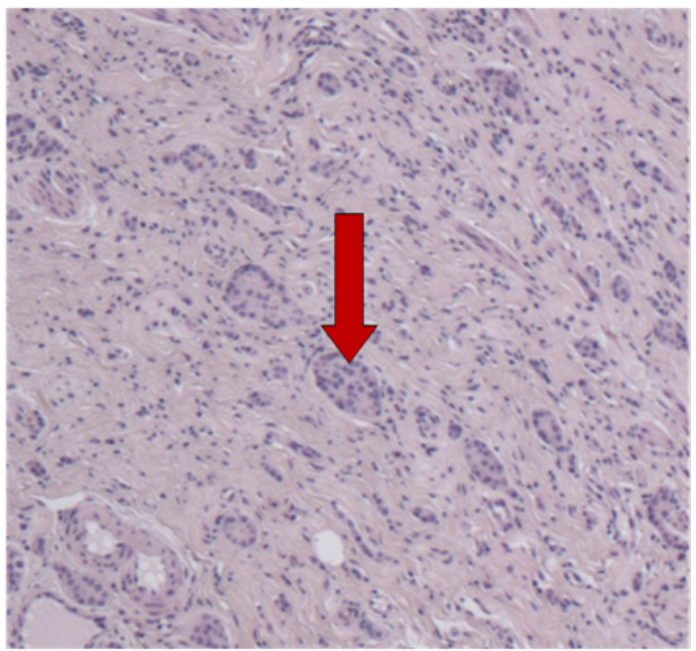

Figure 5 Nests of carcinoid tumour cells (red arrow) infiltrating appendiceal wall. 


\section{Discussion}

Endometriosis is one of the most common diseases seen by gynaecologists. The prevalence is difficult to estimate as women can be asymptomatic or present with varying degrees of pelvic pain. ${ }^{11}$ Endometriosis is associated with infertility and has been identified in $38.5 \%$ of infertile women as opposed to $5.2 \%$ of fertile women. ${ }^{12}$

Appendicular tumours have diverse histology. Carcinoid neoplasms are the most common, accounting for $66 \%$ of all tumours of the appendix. They are comprised of enterochromaffin cells and contain many neurosecretory granules that release serotonin, histamine and prostaglandins. ${ }^{13}$ As with endometriosis, the incidence and prevalence is unknown, as the disease is mainly asymptomatic. The average time for a carcinoid tumour to become symptomatic is 9 years. Carcinoid tumours may present late and be associated with metastasis if found within the tip of the appendix.

The most common benign appendix tumours are mucinous cystadenomas. These tumours are composed of intestinal epithelium are dysplastic and secrete mucin, giving rise to a mucocele. This often causes the appendix to dilate and can present as acute appendicitis. The presence of a mucinous cystadenoma with associated mucin within the appendix wall increases the risk of psuedomyxoma peritonei.

There have been reports in literature of the co-existence of two types of appendicular tumours. Alsaad et al. ${ }^{14}$ reported a case of combined goblet cell carcinoid and mucinous cyst-adenoma of the appendix in a patient presenting with right iliac fossa pain. The occurrence of appendicular tumours co-existing with endometriosis is rarely reported, ${ }^{15}$ and there have been no reported cases of two different appendicular tumours being found with co-existent features of an endometrioma within the same appendix. Appendicectomy specimens should be routinely sent for histopathological analysis. Duzgan et al. ${ }^{15}$ reported an intra-operative detection rate of less than $50 \%$ for all types of appendicular tumours. Jones et al (2007) evaluated the histopathological reports of 1225 appendicectomy specimens. Of the $1225,46(3.75 \%)$ revealed abnormal diagnoses and $24(1.96 \%)$ were clinically significant, altering patient management. ${ }^{16}$ Routine histopathological analysis avoids the potential to miss significant pathologies which may need different treatments.

Currently in the literature there are no clear guidelines regarding the treatment of appendicular tumours. ${ }^{17}$ There is considerable debate as to the use of appendectomy versus right hemicolectomy and the effects on long term results. A right hemicolectomy is indicated with adenocarcinomas, tumours invading the mesoappendix, serosa, lymphatics or vasculature and benign tumours with a diameter of more than $2 \mathrm{~cm} .{ }^{18}$ As preferred by some surgical units ${ }^{19}$ a right hemicolectomy is considered the treatment of choice. There was suspicion of a malignant adenocarcinoma after initial biopsy which rendered a right hemicolectomy a safer option.

\section{Conclusion}

Most surgeons will only encounter a few carcinoid tumours of the appendix in their career. We feel this unique case will add to the small database of similar reports and help with management of this rare disease.

This case illustrates the broad spectrum of appendicular and endometriotic disease. It also demonstrates the role of biopsy of abnormal areas atypical of endometriosis at diagnostic laparoscopy. We hope to highlight the interesting asymptomatic presentation of this patient and therefore the importance of requesting routine histopathological analysis. After prompt treatment with right hemicolectomy, this young woman has fortunately conceived naturally without IVF.

\section{Acknowledgments}

None.

\section{Conflicts of interest}

Author has no any conflict of interest to declare.

\section{References}

1. Benagiano G, Brosens I. History of Adenomyosis. Best Pract Res Clin Obstet Gynaecol. 2006;20(4):449-463.

2. http://www.rcog.org.uk/files/rcog-corp/GTG2410022011.pdf

3. Mounsey AL, Wilgus A, Slawson DC. Diagnosis and management of endometriosis. Am Fam Physician. 2006;74(4):594-600.

4. Remorgida V, Ferrero S, Fulcheri E, et al. Bowel endometriosis: presentation, diagnosis, and treatment. Obstet Gynecol Surv. 2007;62(7):461-470.

5. Heaps JM, Nieberg RK, Berek JS. Malignant neoplasm arising in endometriosis. Obstet Gyncol. 1990;75(6):1023-1028.

6. Slavin RE, Krun R, Van Dinh T. Endometriosis associated intestinal tumours: a clinical and pathological study of 6 cases with a review of the literature. Hum Pathol. 2000;31(4):456-463.

7. Brinton L, Gridley G, Persson I, et al. Cancer risk after a hospital discharge diagnosis of endometriosis. American Journal of Obstetrics and Gyanecology. Am J Obstet Gynecol. 1997;176(3):572-579.

8. Connor SJ, Hanna GB, Frizelle FA. Retrospective clinicopathologic analysis of appendiceal tumours from 7970 appendicectomies. Dis Colon Rectum. 1998;41(1):75-80.

9. Topkan E, Polat Y, Karaoglu A. Primary mucinous adenocarcinoma of appendix treated with chemotherapy and radiotherapy: a case report. Tumori. 2008;94(4):596-599.

10. Azordegan N, Yazdankhah A, Moghadasian MH. A rare case of coexistence of carcinoid tumour of appendix vermicularis and ileal endometriosis. Arch Gynecol Obstet. 2009;279(2):183-187.

11. Slaughter K, Gala RB. Endometriosis for the colorectal surgeon. Clin Colon Rectal Surg. 2010;23(2):72-79.

12. Verkauf BS. Incidence, symptoms and signs of endometriosis in fertile and infertile women. J Fla Med Assoc. 1987;74(9):671-675.

13. Robertson RG, Geiger WJ, Davis NB. Carcinoid tumours. Am Fam Physician. 2006;74(3):429-434.

14. Alsaad KO, Serra S, Chetty R. Combined goblet cell carcinoid and mucnious cystadenoma of the vermiform appendix. World J Gastroenterol. 2009;15(27):3431-3433.

15. Duzgan AP, Moran M, Uzun S, et al. Unusual findings in appendicectomy specimens: Evaluation of 2458 cases and review of the literature. Indian $J$ Surg. 2004;66(4):221-226.

16. Jones AE, Phillips AW, Jarvis JR, et al. The value of routine histopathological examination of appendicectomy specimens. BMC Surg. 2007;7:17.

17. Carr NJ, Sobin LH. Unusual tumours of the appendix and pseudomyxoma peritonei. Semin Diagn Pathol. 1996;13(4):314-325.

18. Modlin IM, Sandor A. An analysis of 8305 cases of carcinoid tumours. Cancer.1997;79(4):813-829.

19. Cortina R, McCormick J, Kolm P, et al. Management and prognosis of adenocarcinoma of the appendix. Dis Colon Rectum. 1995;38(8):848-852. 